\title{
A LABORATORY DEMONSTRATION OF HIGH-RESOLUTION HARD X-RAY AND GAMMA-RAY IMAGING USING FOURIER-TRANSFORM TECHNIQUES
}

David Palmer and Thomas A. Prince

California Institute of Technology, Pasadena, CA 91125

\section{Abstract}

A laboratory imaging system has been developed to study the use of Fourier-transform techniques in high-resolution hard $\mathrm{x}$-ray and $\gamma$-ray imaging, with particular emphasis on possible applications to high-energy astronomy. We discuss considerations for the design of a Fourier-transform imager and describe the instrumentation used in the laboratory studies. Several analysis methods for image reconstruction are discussed including the CLEAN algorithm and maximum entropy methods. Images obtained using these methods are presented.

\section{Introduction}

It has been recognized for some time that Fourier-transform techniques can in principle be used to achieve arc-second imaging capability at $\mathrm{x}$-ray and hard $\mathrm{x}$-ray energies. Imaging in Fourier space can take many forms [1-6] and has application in numerous fields. We will concentrate in this paper on implementation of a technique suitable for high-energy astronomy which has been discussed by Makishima et al. [3] and Hurford and Hudson [5].

Our objectives in this particular study were relatively narrow in scope. We wished to investigate the practical implementation of one specific form of the Fourier-transform technique in the context of development of a high-resolution hard x-ray and $\gamma$-ray telescope for solar flare observations. In the type of Fourier-transform imaging considered here, the source region is viewed simultaneously through multiple apertures, each of which provides information about one Fourier component of the source distribution. Each individual aperture consists of a pair of widely separated collimator grids which form a modulation pattern of incident $\gamma$-rays on appropriate photon detectors. This concurrent multi-grid technique has distinct advantages for the study of solar flares since it allows instantaneous photon-limited images to be formed, in contrast to time modulation Fourier-transform techniques in which the time needed to obtain a complete image is determined by the time scale of the modulation.

A multi-grid x-ray and hard x-ray Fourier-transform imager has been part of the NASA Pinhole Occulter Facility concept [7]. We have been interested in extending the energy range of such an instrument into the $\gamma$-ray regime, well above $100 \mathrm{keV} \mathrm{[8].} \mathrm{Since}$ the Fourier-transform technique has not been widely applied at $\mathrm{x}$ ray energies or at $\gamma$-ray energies, we were interested in exploring practical systematic effects that may limit the effectiveness of imaging, and also in exploring the practical constraints placed on an imaging system by conventional photon detection and collimation techniques.

There are numerous approaches to high-energy photon imaging in Fourier-space other than the specific technique discussed in this paper. We refer the reader in particular to several earlier references that deal with the implementation and simulation of Fourier-transform imagers in the laboratory [9-12].

\section{Description of Method}

\section{Basic Technique}

In the multi-grid Fourier-transform technique considered here, pairs of widely separated collimator grids are used to form modulation patterns of incident $\gamma$-rays that can be measured by $\gamma$-ray detectors with moderate spatial resolution. The collimator grids consist simply of slits with a certain orientation and a certain spacing $S$. The top and bottom grids are separated by a distance $D$ and differ slightly in $S$, producing a characteristic triangular modulation pattern of incident photons at the detector (see Figure 1). Each pair of collimator grids allows the measurement of the phase and amplitude of a particular Fourier component of the source angular distribution with angular period $S / D$ (assuming a small angle approximation). Specifically, the Fourier component of the source distribution is related to the event position distribution by [5]:

$$
\int_{\theta_{\min }}^{\theta_{\max }} F(\theta) e^{i \frac{\pi D}{S} \theta} d \theta=\frac{\pi}{4 \in A t} \int_{\frac{-P}{2}}^{\frac{P}{2}} N(x) e^{i \frac{2 \pi}{P} x} d x
$$

where $F(\theta)$ is the angular source distribution, $\theta$ is the angle along the sensitive direction of the collimator grids, $\theta_{\max }$ and $\theta_{\min }$ define the boundaries of the field of view, $\epsilon A$ is the effective detector area, $t$ is the observation time, $P$ is the period of the triangular modulation pattern, and $N(x)$ is the idealized triangular count distribution. The phase and amplitude information from the individual grid-pairs with different spatial periods and orientations is combined to form images using algorithms similar to those employed in radio astronomy.

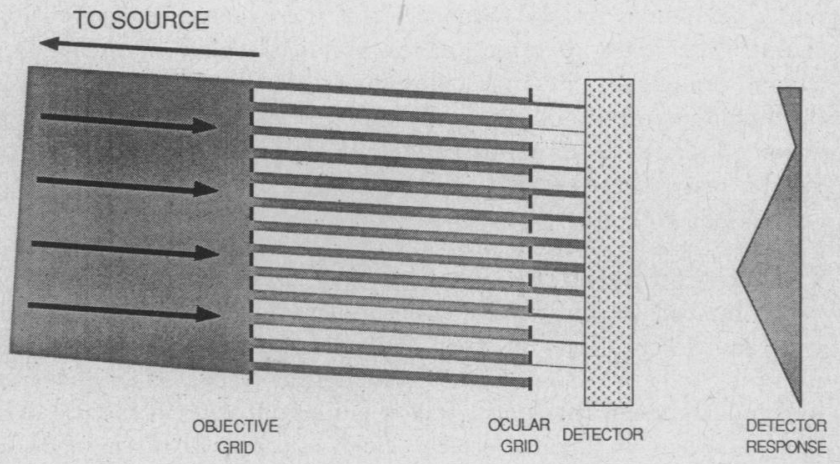

Figure 1. Illustration of the Fourier-transform imaging technique. A parallel beam of photons incident on a pair of collimator grids forms a modulation pattern at the detector which is smoothed by the detector spatial resolution. 


\section{Design Considerations}

Number of Grids. A basic choice in the design of an imag ing system involves specification of the number of Fourier components to be measured. The number of Fourier components measured is related directly to image completeness. A larger number allows more complete coverage of Fourier space and consequently a more detailed image. In most practical imaging systems the coverage in Fourier space is quite sparse and rarely approaches the sampling theorem limit.

The requirement on the number of Fourier components, and therefore on the number of apertures, is a complex issue outside the scope of this paper and depends critically on the complexity of the source region as well as on the contrast of the features within the source region. Very crudely, the greater the number of distinct sources within the field-of-view, the greater the number of Fourier components that must be measured. Since each distinct source has a characteristic strength and size scale, as well as a position, a minimum of four parameters must be estimated from the measured amplitudes and phases of the Fourier components. A further consideration is that sufficient sampling of the Fourier components must occur over any octave of spatial frequencies in which there is possible spatial structure. For our laboratory tests we measured up to 84 components for a field of view containing 3 sources. However, a significant fraction of the measured components were redundant, providing primarily a consistent phase calibration (see below).

Detector Resolution. For a detector of a given size and spatial resolution, there is a tradeoff between the number of Fourier components measured, and the degree of sampling of each component. The degree of sampling of the modulation pattern determines the signal-to-noise ratio of the amplitude and phase determination and the degree of redundancy and background rejection. The requirement on the degree of sampling is readily quantified. At least 4 samples of each modulation pattern are needed to achieve a signal-to-noise ratio that is $90 \%$ of the optimum [5]. In our laboratory studies, one period of the modulation pattern corresponded to about 5.5 times the FWHM resolution of the detector giving us at least 5 samples of each modulation pattern.

The finite detector resolution also places a practical restriction on the idealized measurement of Fourier amplitude and phase considered in equation (1). In principle, source components with angular frequency higher than $D / S$ could be measured with the system if the higher spatial frequencies of the count rate distribution could be detected. In practice, the amplitude of these higher frequency components is much reduced compared to the amplitude of the fundamental modulation. Furthermore, the finite spatial resolution of the detector tends to smooth the spatial distribution of events, restricting the measurement in practice to the largest spatial period, $P$.

Grid Spatial Frequencies and Orientations. Once the total number of grid-pairs has been specified, the spatial frequencies and orientations of the grid-pairs need to be chosen. A natural choice is a logarithmic distribution of spatial frequencies, particularly when the source region is likely to have structure over a wide range of angular scales. Such a choice is often made in radio astronomy for complex source regions.

The orientations of the grids should be quasi-random, that is, they should avoid oversampling of any given angular range, providing approximately uniform sampling over spatial frequencies. In the laboratory imaging system we used logarithmic grid spacings but deviated from the optimal choice of quasi-random orientations to simplify the design of the multi-grid system. In particular, certain angles were oversampled. The implications of this design choice will be discussed below.

\section{Experimental Setup}

The basic experimental setup is shown in Figure 2 and consisted of a set of 7 collimator-grid pairs and a $\mathrm{NaI}$ scintillation camera. The grids consisted simply of lead (solder) wire of varying diameter stretched across frames. The objective grids had a length perpendicular to the wire direction of $35 \mathrm{~mm}$ and a width of 28 $\mathrm{mm}$. The grid periods (i.e. wire spacings) are given in Table I together with the wire diameter, the number of cycles in the modulation pattern at the detector, and the spatial period measured at the source. The difference in wire spacing between the ocular and objective grids was determined by the requirement of an extra period in one of the two grids in each pair and by the geometric convergence caused by the finite source distance $(2.0 \mathrm{~m}$ from the objective grid).

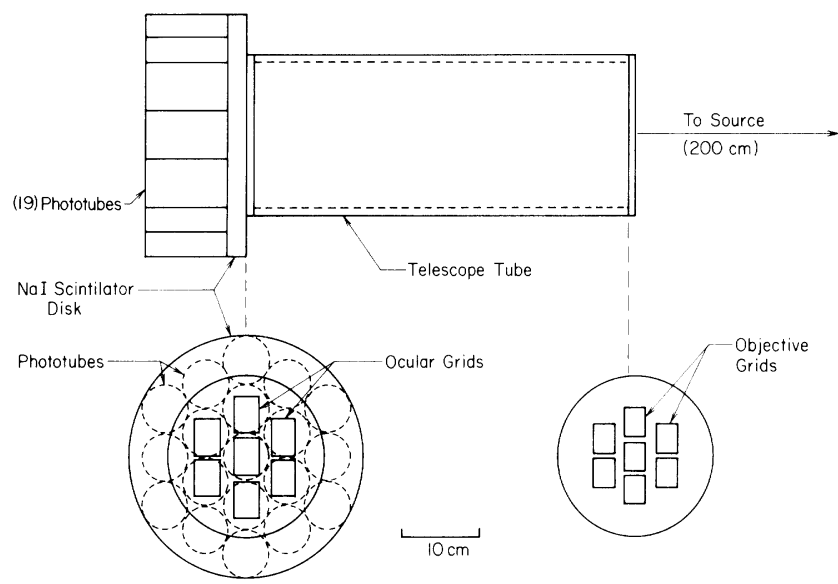

Figure 2. Schematic diagram of the laboratory imaging system.

We note that the grids were rather coarse. Future telescopes will use grids with periods of $100 \mu$ or better, a factor of ten finer than those employed in this initial laboratory study.

All 7 grids used in the laboratory setup had the same orientation. In order to obtain a sufficient number of different angular orientations, we mounted the sources on a bearing and rotated them around the axis of the telescope. Up to 12 orientations were sampled in the laboratory imaging studies.

The spatial periods of the grids were logarithmically spaced by a factor of approximately the third root of two to insure that the periods of components in the same octave of spatial frequencies were relatively incommensurate. This is particularly important since all grids had the same orientation.

The $\mathrm{NaI}$ scintillation camera was $1.3 \mathrm{~cm}$ thick and $30 \mathrm{~cm}$ in diameter. It was read out with the "Anger camera" technique using 19 two-inch diameter photomultiplier tubes which were simultaneously pulse-height analyzed. The pulse-height data were encoded on magnetic tape for subsequent analysis and event position determination. A position resolution of $1.7 \mathrm{~mm} \mathrm{rms}$ was obtained at the $122 \mathrm{keV}$ line of ${ }^{57} \mathrm{Co}$. This same line energy was used for all images shown in this paper.

\begin{tabular}{|c|c|c|c|c|c|c|}
\hline \multicolumn{7}{|c|}{ Table I } \\
\hline \multirow{2}{*}{ Grid } & \multicolumn{2}{|c|}{ Objective } & \multicolumn{2}{|c|}{ Ocular } & \multirow{2}{*}{$\begin{array}{c}\text { Number } \\
\text { of } \\
\text { Cycles }\end{array}$} & \multirow{2}{*}{$\begin{array}{c}\text { Period } \\
\text { at Source } \\
(\mathrm{mm}) \\
\end{array}$} \\
\hline & $\begin{array}{c}\text { Spacing } \\
\text { (mm) }\end{array}$ & $\begin{array}{l}\text { Wire Dia. } \\
(\mathrm{mm})\end{array}$ & $\begin{array}{c}\text { Spacing } \\
\text { (mm) }\end{array}$ & $\begin{array}{c}\text { Wire Dia. } \\
(\mathrm{mm})\end{array}$ & & \\
\hline 0 & 1.08 & 0.64 & 1.27 & 0.64 & 1.30 & 5.29 \\
\hline 1 & 1.27 & 0.64 & 1.69 & 0.81 & 1.63 & 6.35 \\
\hline 2 & 1.89 & 0.81 & 1.95 & 1.02 & 1.63 & 8.47 \\
\hline 3 & 2.12 & 1.02 & 2.42 & 1.27 & 1.46 & 10.58 \\
\hline 4 & 2.54 & 1.27 & 2.82 & 1.80 & 1.63 & 12.7 \\
\hline 5 & 3.39 & 1.60 & 3.63 & 2.38 & 1.63 & 16.9 \\
\hline B & 4.23 & 2.38 & 4.42 & 2.36 & 1.54 & 21.2 \\
\hline
\end{tabular}




\section{Analysis Methods}

The analysis methods used are similar to those employed in radio astronomy to reconstruct an image from an undersampled set of Fourier components of the source distribution. While there is a close analogy between high-energy photon Fourier-transform imaging and conventional radio-interferometry, an important practical difference between the two techniques is the typical signal-to-noise ratio and statistical fluctuations in each of the methods. In radiointerferometry, the quantum limit is not usually a consideration, while the treatment of systematic errors is a significant issue. In contrast, multi-grid Fourier-transform techniques were developed for application in high-energy photon imaging where photon statistics and background fluctuations are often limiting factors. These statistical considerations play an important role in two areas: determination of amplitude and phase from individual grid-pairs, and formation of an image given measurements from several gridpairs.

A further difference between radio-astronomy techniques and the multi-grid Fourier imaging technique discussed here is the smaller number of components generally measured in the latter technique. Typically, fewer than 100 components have been measured in our studies, making computational techniques such as fast Fourier transforms unnecessary. However, considerations such as image fidelity and completeness of sampling become correspondingly more important when fewer components are measured.

Fourier Amplitude and Phase Determination. The first step in forming the image is the determination of the amplitudes and phases and their respective errors. Because the spatial count distribution due to a point source is ideally a triangle with a peak position linearly related to the phase, we may consider each event to be a measurement of the phase of the source distribution filtered by a triangular response function. It is convenient to consider the measurement process as a random walk in the complex plane. Each event corresponds to a step of uniform length with direction (i.e. phase) given by detector position. Since the phase is linearly related to detector position, we will consider the detector position to be directly calibrated in terms of phase, $\phi$, and have a range of $(0-2 \pi)$. The end point of the random walk is then given by:

$$
A e^{i(t)}=\sum_{\text {events }} e^{i \phi_{j}} \quad(A, \Phi \text { real }),
$$

where $\phi_{j}$ is the measured position of the jth event calibrated in phase units, and the sum is over all events. The random walk amplitude $A$ and phase $\Phi$ are directly related to the amplitude and phase of the respective Fourier component of the source distribution via equation (1), since the sum in equation (2) is the discrete event realization of the integral over the count distribution in equation (1).

It is important to determine the statistical error in the phase and amplitude measurements and incorporate this information in the image reconstruction algorithms. The random walk description is particularly convenient since errors may be calculated in the usual manner for a random walk process.

Given the measurement of the Fourier amplitudes and phases and the corresponding errors, images have been reconstructed using one of two methods, both common in radio-astronomy: Fourier reconstruciion followed by application of the CLEAN algorithm [13] and the Maximum Entropy Method (MEM)[14,15]. Below, we will summarize the relative merits of the two methods for application to high-energy photon imaging. More complete specification of the methods are given in the indicated references.
Phase Calibration. An important issue in our laboratory studies was calibration of phases. As mentioned, in order to obtain a sufficient number of grid orientations, the sources were rotated within the field of view of the telescope. Because we rotated the sources and not the telescope (for reasons of mechanical simplicity) and because of the slight difference between the axis of rotation and the telescope axis, it was necessary to calibrate the telescope for each image. The calibration consisted of finding the phase of each grid's modulation pattern at a known point, most conveniently the center of rotation of the source. We emphasize that such a procedure would not be necessary for a solarastronomical telescope with quasi-random grid orientations. Such a telescope could be calibrated once with a point source and would remain in calibration as long as it kept its dimensional stability.

The phase calibration procedure involved iteratively comparing the phase determined from all but one grid-pair with the direct measurement of phase from the omitted grid-pair and requiring consistency. This procedure worked well in practice as evidenced by the images in Section 3. However, the procedure is less than ideal in that a significant fraction of the data is used to insure a consistent phase calibration. Future laboratory studies will attempt to determine the source-telescope reference through use of an optical alignment system that is independent of the $\gamma$-ray measurements.

Fourier Reconstruction and CLEAN Algorithms. In this method the measured Fourier components are combined with their respective phases and amplitudes to produce an "uncleaned" image. This preliminary image generally has large sidelobes, particularly if there is one or more dominant point source of emission in the field of view. The CLEAN algorithm attempts to reduce these sidelobes by iteratively deconvolving the image with the expected response of an ideal point source including sidelobes. This method has been shown to be effective for a wide variety of source configurations, although the method works better for collections of point sources than for diffuse emission regions. The advantages of the CLEAN method are that it is relatively fast and theoretically simple to apply. Some disadvantages, particularly in our application, are that the method can produce images with areas of negative pixel brightness, that statistical errors are somewhat difficult to incorporate, particularly if the errors vary from component-to-component, and that significant sidelobes can still be present in the image, particularly if the statistical errors are large.

Maximum Entropy Method. Considerable literature exists concerning the implementation and rationale for the maximum entropy method (see references cited above). Basically, the method attempts to produce the "smoothest" (highest entropy) image compatible with the data. Advantages of the method are that all image pixels have positive brightness, and that the statistical errors of individual Fourier components are easily incorporated into the analysis. While the maximum entropy method has a bias towards diffuse sources of emission, in practice the method works well even for point sources, producing images with lower sidelobes and other artifacts than the CLEAN algorithm. The comparison of the two methods will be illustrated in the results presented in the next section. 


\section{Results}

Figures $3 \mathrm{a} \& 3 \mathrm{~b}$ show images of a single ${ }^{57} \mathrm{Co}$ source taken with the laboratory imaging system. Figure $3 \mathrm{a}$ is a contour plot of inferred intensity while Figure $3 \mathrm{~b}$ is a gray scale plot of the same image. The field of view is $60 \mathrm{~mm} \times 60 \mathrm{~mm}$. Approximately 600 photons were detected in each Fourier component with about equal contributions from source and background. The data set for the images included six angular orientations.

The images in Figure 3 were reconstructed using the maximum entropy method. While the source is clearly visible, the background has linear artifacts which are particularly apparent in the gray scale image. These artifacts are the result of the oversampling of angles resulting from the limited number (6) of angular orientations in the data set.

Figures $4 \mathrm{a}$ and $4 \mathrm{~b}$ show images of three ${ }^{57} \mathrm{Co}$ sources obtained using the CLEAN and the maximum entropy methods respectively.

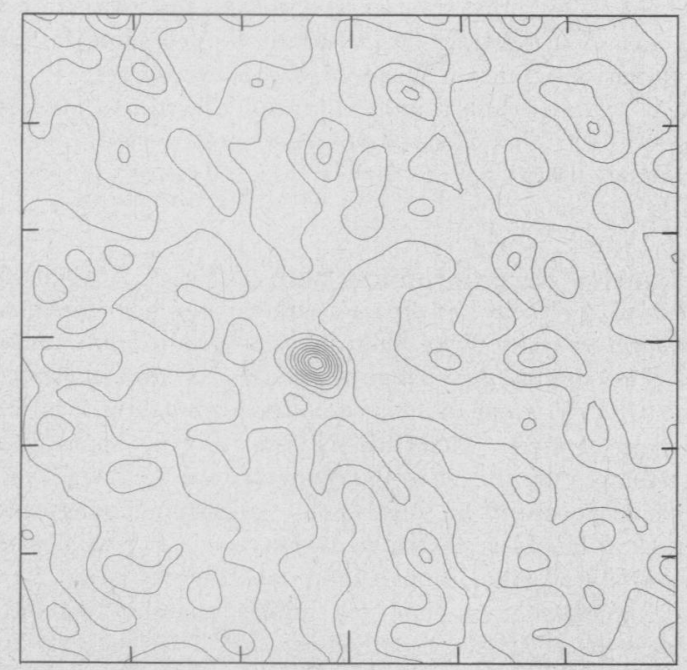

Figure 3a. Contour plot of the image of a single ${ }^{57} \mathrm{Co}$ source at $122 \mathrm{keV}$ reconstructed using maximum entropy methods. Contours are at $10 \%$ intervals of the dynamic range of intensities. The field of view is $60 \mathrm{~mm} \times 60 \mathrm{~mm}$.

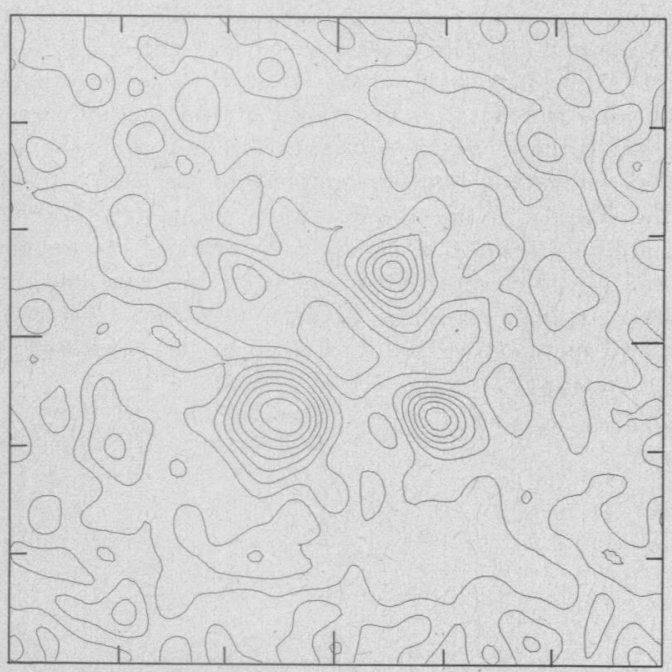

Figure 4a. Contour plot of the image of three ${ }^{57} \mathrm{Co}$ sources produced using the CLEAN algorithm. Contours and size of field of view are the same as in Figure 3a.
Each source field is $60 \mathrm{~mm} \times 60 \mathrm{~mm}$. The radioactive sources used to make these images were not point sources, rather they had widths up to about $7 \mathrm{~mm}$. The exact spatial profile of the activity of the sources was uncalibrated. In fact, the images in Figure 4 provide our current best estimate of the spatial extent of the sources. Future images with spatially calibrated sources are planned.

Both images in Figure 4 were reconstructed from the same data set which included 12 angular orientations. Note the considerably smaller level of artifacts and sidelobes in the maximum entropy image compared to the CLEAN image.

Figures 5 and 6 give different presentations of the maximum entropy image of Figure $4 \mathrm{~b}$ which bring out different details of the source profiles and the background artifacts. Figure 5 is a gray scale plot of the maximum entropy image while Figure 6 is a 3 dimensional contour plot of the same image.

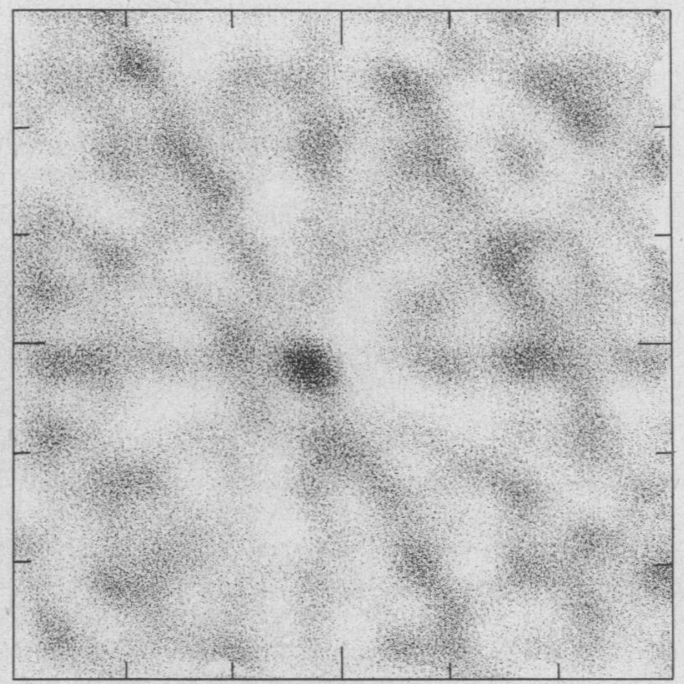

Figure 3b. Gray scale plot of the maximum entropy image shown in Figure 3a. Note the linear artifacts due to the limited number of angular orientations sampled.

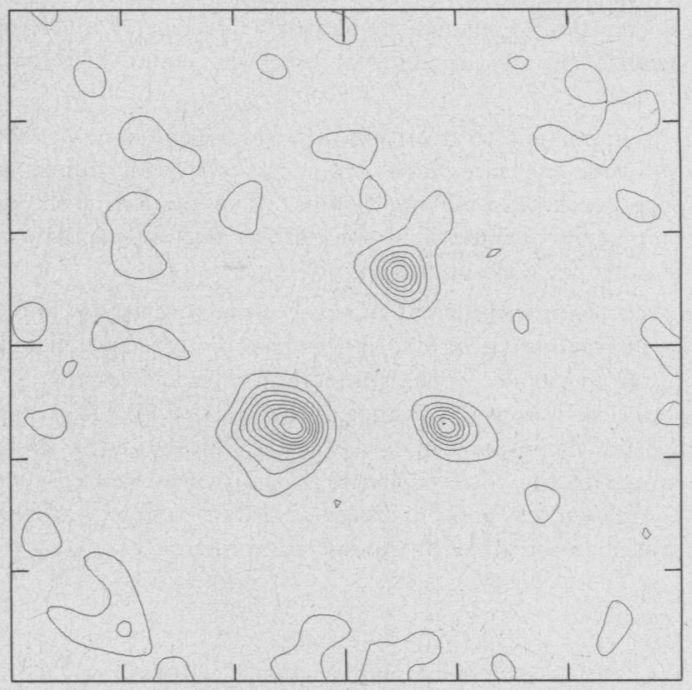

Figure 4b. Contour plot of the image of three ${ }^{57} \mathrm{Co}$ sources produced using the maximum entropy method. The data set for the image was identical to that used to produce Figure $4 \mathrm{a}$. 


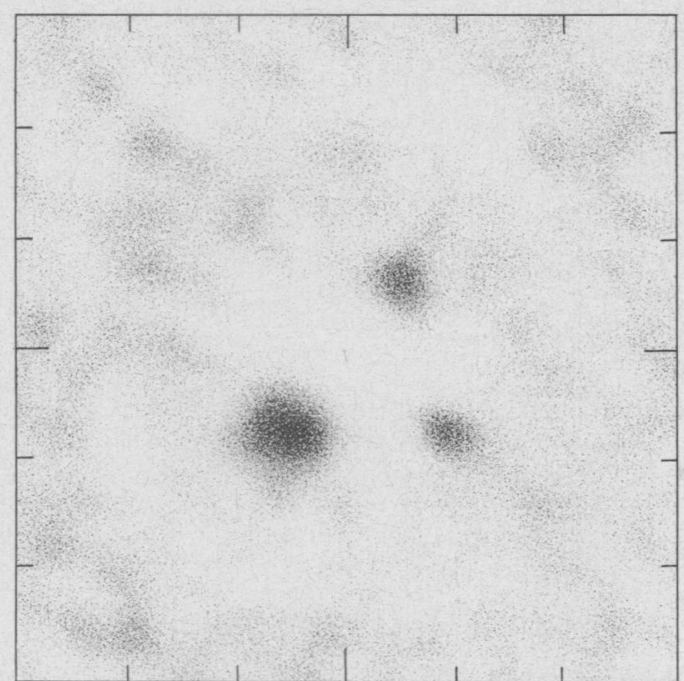

Figure 5. Gray scale plot of the maximum entropy image shown in Figure $4 \mathrm{~b}$.

\section{Discussion}

Our initial laboratory studies using conventional gamma-ray detectors and simple grid structures indicate the feasibility of multi-grid Fourier-transform techniques for hard $x$-ray and $\gamma$-ray imaging. We have investigated both CLEAN algorithms and maximum entropy methods for image reconstruction. In our studies, the maximum entropy method produced superior results, primarily because of its ability to incorporate the statistical errors in individual phase and amplitude measurements, leading to improved suppression of background artifacts. Future simulations and laboratory studies will explore the issues of image complexity and dynamic range using the CLEAN and maximum entropy reconstruction methods.

As mentioned in the introduction, our studies were undertaken in the context of development work for a high-resolution solar hard $x$-ray and $\gamma$-ray imager. While several systematic effects proved to be important in the laboratory studies, principally phase inter-calibration and oversampling of angular orientations, these were specific to the laboratory studies and do not pose problems for an astronomical telescope.

A significant difference between the laboratory imaging system described here and an eventual solar-astronomical telescope is the much finer angular resolution anticipated for the latter. In these laboratory studies, the resolution was limited by the coarseness of the grids to $5.3 \mathrm{~mm}$ at $2.5 \mathrm{~m}$, or somewhat better than 7 minutes of arc. Much finer, arc-second resolution will be achievable using $50 \mu$ grid periods and a $10 \mathrm{~m}$ grid separation.

We emphasize the preliminary nature of these initial studies. Among the future improvements contemplated are: use of finer grids with spacings down to $100 \mu$ or better, an optical alignment system to provide an independent measurement of the source positions, and spatially calibrated sources of various configurations to probe more quantitatively the imaging characteristics of the laboratory system. In addition, considerable work remains to be done in simulating the imaging of complex source regions and in determining the relative merits of various image reconstruction algorithms.

We acknowledge useful discussions with several members of the NASA Pinhole Occulter Facility Science Working Group and the NASA Max '91 Science Study Group, in particular Gordon Hurford. This work was supported in part by NASA Grant NAG8-588 and NSF Grant AST-8351736, part of the NSF PYI program.

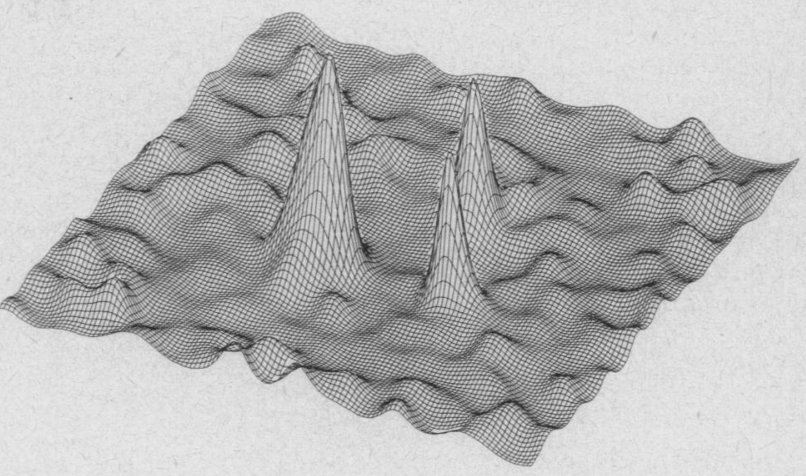

Figure 6. Three-dimensional contour plot of the maximum entropy image shown in Figure $4 \mathrm{~b}$ and Figure 5.

\section{References}

[1] L. Mertz, Transformations in Optics. New York: John Wiley \& Sons, Inc., 1965.

[2] L. Mertz, "Applicability of the Rotation Collimator to Nuclear Medicine," Opt. Commun., 12, p. 216, 1974.

[3] K. Makishima, S. Miyamoto, T. Murakami, J. Nishimura, M. Oda, Y. Ogawara, and Y. Tawara, "Modulation Collimation as an Imaging Device," in New Instrumentation for Space Astronomy, van der Hucht and Vaiana (eds.), New York: Pergammon, 1977 , p. 277.

[4] C. Chou and H.H. Barrett, "Gamma-ray Imaging in Fourier Space," Optics Letters, 3, p. 187, 1978.

[5] G. J. Hurford and H. S. Hudson, "Fourier-Transform Imaging for X-ray Astronomy," UCSD-SP-79-27 and BBSO preprint $0188,1979$.

[6] H. H. Barrett and W. Swindell, Radiological Imaging (Vol. 2). New York: Academic Press, 1981.

[7] E. A. Tandberg-Hanssen, H. S. Hudson, J. R. Dabbs, and W. A. Baity (eds.), "The Pinhole/Occulter Facility," NASA Technical Paper 2168, 1983.

[8] C. J. Crannell, G. J. Hurford, L.E. Orwig, and T. A. Prince, "A Fourier Transform Telescope for Sub-arcsecond Imaging of X-rays and Gamma Rays," Proc. SPIE, 571, p. 142, 1986. (See also "Report of the Max '91 Science Study Group", NASA, 1986.)

[9] C. Chou, Coded Imaging Systems Using a Fourier Aperture. Ph.D. Thesis: University of Arizona, 1978.

[10] L. Renaud, M. L. G. Joy, and D. L. Gilday, "Fourier Multiaperture Emission Tomography," J. Nucl. Med., 20, p. 986, 1979.

[11] C. Chou, "Fourier Coded Aperture Imaging in Nuclear Medicine," SPIE, Application of Optical Instrumentation in Medicine XII, 454, p. 457, 1984.

[12] P. K. H. Wang and M. L. G. Joy, "Quantum Noise Measurements for Fourier Multiaperture Emission Tomography," IEEE Trans. Med. Imaging, MI-4, p. 129, 1985.

[13] J. A. Hogbom, "Aperture Synthesis with a Non-regular Distribution of Interferometer Baselines," Astron. Astrophys. Supp., 15, p. 417, 1974.

[14] S. F. Gull and G. J. Daniell, "Image Reconstruction from Incomplete and Noisy Data," Nature, 272 , p. 686, 1978.

[15] T. J. Cornwell and K. F. Evans, "A Simple Maximum Entropy Deconvolution Algorithm," Astron. Astrophys., 143, p. $77,1985$. 